\title{
Ses ThedTLAS
}

\section{Biomimetic Engineering Analysis of Heliotropic Plants}

Jerome Moore and Adam Hines, Raytheon Corporation and Texas Tech University, Lubbock Texas, 79409, Email: Jerome.Moore@TTU.edu and Adam.Hines@TTU.edu

Received 27 June 2017; Revised 3 July 2017; Accepted 5 July 2017

Copyright (C)2017 Jerome Moore and Adam Hines. This is an open access article distributed under the Creative Commons Attribution License (https://creativecommons.org/licenses/by/4.0/), which permits unrestricted use, distribution, and reproduction in any medium, provided the original work is properly cited.

Available online 7 July, 2017 at www.atlas-journal.org, doi: 10.22545/2017/00085

$\mathrm{B}$ iomimetics is the field of study that identifies potential useful biological processes and mechanisms in nature and translates these principles into the engineering domain. Utilizing natures abilities has proven to be a powerful and useful mechanism for innovation, and has led to profound technical advancements and capabilities in applied science disciplines and fields. The main objective of this paper is to introduce a Biomimicry analysis of the plants ability to maintain optimal sunlight in order to maximize the photosynthesis process. The main components and factors that enable heliotropism are discussed as well as the governing physics describing the processes needed in order for this phenomenon to occur in nature. The reader should emerge from this paper with an understanding of how plants are induced by sunlight as well as the long-term possibility of replicating this phenomenon for human purposes.

Keywords: Biomimetics, Biomimicry, Heliotropism, solar tracking, transdisciplinary research.

\section{Introduction}

In a time when energy costs continue to increase and concerns associated with an over dependence on fossil fuels, it is advantageous for engineers and companies to capitalize on the technological advances and capabilities that allow us to replicate nature's approach in harvesting energy. Biomimicry is leading the approach to innovation by seeking sustainable solutions to human challenges by emulating nature's time-tested patterns and strategies [1]. Biomimetic engineering is an interdisciplinary approach that identifies potential useful processes and mechanisms in biological systems and imitates them in engineering systems [2]. This engineering approach is initiated by identifying and analyzing a solution offered by nature that may translate to an engineering problem. The underlying principles are understood and transferred to the artificial realm through design, simulation, and fabrication. If possible, affordances and improvements are made within the confines of the technological state [3]. Using the Biomimetic Engineering approach, a common phenomenon that occurs with plants obtaining the most optimal amount of sunlight will be examined, and this process will 
be considered to provide viable solutions that may produce cost-effective renewable energy for human purposes.

First, a common ground must be established for photosynthesis and the effect heliotropism has in enhancing its efficiency. This must be discussed to show its importance to everyday life and its ability to maximize a process that ensures our ability to live on earth. In grade school, the science curriculum commonly teaches that photosynthesis is responsible for supplying all of the earth's organic compounds as well as the energy required to sustain life on the planet. This process is a complex biological process that harvests light energy from the sun and converts it into biological energy [4]. The energy and carbon is transmitted throughout the ecosystem as the photosynthetic organisms are consumed or decomposed by other organisms in a continuous, revolving circle of life. The principle inputs on the rate of photosynthesis are available water, light wavelength and intensity [5]. However, the main factor that often gets overlooked is how plants ensure they obtain enough sunlight. Sunlight makes up 2 of the $3 \mathrm{im}-$ portant factors in the photosynthesis process. The process responsible for obtaining the precise amount of ideal sunlight for photosynthesis to occur is called heliotropism.

As stated previously, heliotropism enables plants to maintain an efficient level of sunlight in order to initiate the photosynthesis process. However, just knowing this definition at the surface level does nothing to properly quantify the enormity of this capability's impact on the world. Therefore, a number of components and questions need to be addressed in order to fully comprehend this complex everyday process. First, heliotropism is defined as the directional motion of plant parts in response to the direction of the sun [6]. There are several questions that automatically arise when you contemplate the dynamics of a flower tracking and moving in response to the sun. The major questions would be the following:

- How do plants detect the sunlight?

- What mechanisms enable the plant to track the sun?

- What plant features enhance its heliotropism capabilities?

- What are the fundamental physics involved in the process?
The all-encompassing question is, "Can the plant's heliotropism principles be translated by Biomimetic engineering to innovate or improve upon available technologies/strategies employed to harvest renewable energy?" The main objective of this paper is to address the questions above by introducing a Biomimetic Engineering analysis of the plants' ability to maintain optimal sunlight in order to maximize the photosynthesis process. The main components and factors that enable heliotropism are discussed as well as the governing physics describing the processes needed in order for this phenomenon to occur in nature. The reader should emerge from this paper with an understanding of how plants are induced by sunlight as well as the long-term possibility of replicating the principles of this phenomenon for human purposes.

\section{Observation: The Plant's Ability to Maintain Optimal Sunlight}

Science has ingrained the principle that plants require the sun to grow. However, not all plants require the same amount of sun in order to reproduce or grow. Certain types of plants have heliotropic characteristics to ensure they can initiate photosynthesis due to climatic factors. The most common types of heliotropic plants come from the legumes family; whereas the most known heliotropic flowers are Sunflowers as seen in Figure 1, Poppies, Marigolds, Daisies, and the Snow Buttercup [4]. Other commonly known plants that show heliotropic abilities are alfalfa and cotton [5]. Although, a distinction needs to be made between heliotropic plants and phototrophic plants which are closely related terms. Phototropism is a higher level function in which organism respond to a light stimulus. For example, when a potted plant is placed on a window sill, the plant will be stimulated to lean towards the window in order to obtain more sunlight and increase its growth capabilities. Heliotropic plants follow the direction of the sun throughout the day. Plants exhibiting heliotropic capabilities are not only stimulated by the sun, but physically track the sun's motion across the sky from east to west [6].

The first question tends to focus on how heliotropic plants can move without muscles. The easiest answer is that they have genes that work to an "internal" clock that corresponds with the sun's movement. Scientists at the University of California Davis, de- 


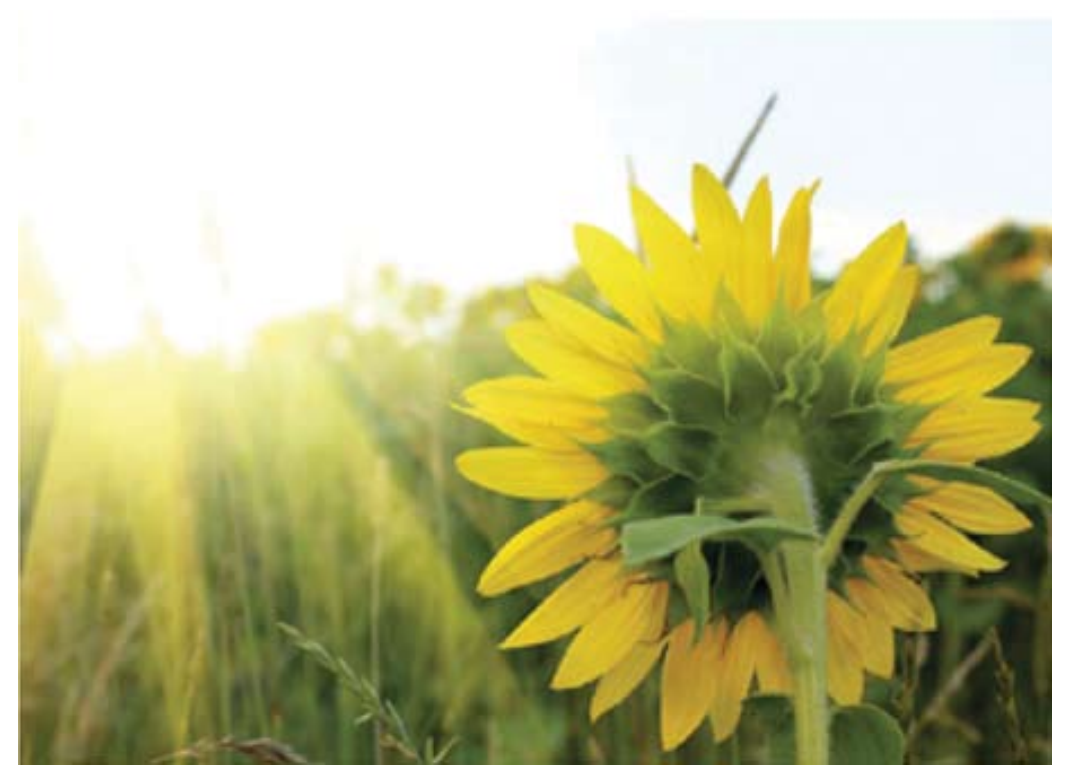

Figure 1: The most commonly known heliotropic plant, the sunflower.

termined that genes stimulate growth on the west side of the stems during the evening hours when the sun is down in preparation of dawn. As a result, the heads/leaves of the plant orient east. When the sun rises, growth is directed to the eastern portion of the stems at a rate that correlates with the movement of the sun throughout the day. This growth rate is calibrated to a precision that enables the plants to "track" the sun to obtain the most amount of sunlight [7]. After the sun has set again, some heliotropic plants assume a random orientation; whereas others enable chemical reactions to return the leaf or flower back to the east facing orientation in preparation for dawn. The movement and gene stimulation can be attributed to the pulvinus or flexible sections of the plant that change the pressure of localized tissues by producing increased levels of potassium ions [8].

There have been numerous hypothesis as to why these plants have this capability ranging from offensive tactics to attract pollinators all the way to a polar opposite theory that attributes this mechanism as a sort of a self-defense mechanism to ensure its survival. While some of these hypotheses may contradict, scientists have reason to believe all the theories may be correct. That in fact this phenomenon is a benefit to not only the plant, but to all that come into direct and indirect contact with the plants. The three most common hypotheses will be discussed in the following paragraphs.

\subsection{The Heating Hypothesis}

This theory focuses on a mutualism relationship in which both pollinators and plants benefit by the heating of the sun. The heat energy absorbed by the plant enhances seed production. Generally, pollinators do not have the ability to generate their own heat and rely on the sun's heat to warm them. Therefore, heliotropic plants are attractive to these pollinators because they tend to be the warmest plants in any given habitat because of their optimal alignment with the sun at all day time hours. This warming effect provides the pollinators with a comfortable environment in which they can warm themselves and/or feed off the nectar. Pollination then occurs which leads to the production of seeds [9]. The pollinator attraction hypothesis is a direct reward for pollinators and the heliotropic plants [10].

\subsection{The Growth Hypothesis}

The growth hypothesis assumes that effective absorption of solar energy and the resulting rise in temperature has an effect on pollen germination and the growth of the pollen tube and seed production [11]. At first glance, this hypothesis seems similar to the heating mutualism theory; however, the growth hypothesis simply states that the plants have this ability for growth purposes only. This is not a mutualism capability in which nature developed to benefit both the pollinator and the plant. This hypothesis believes the pollinator role is attributed to evolution 
in which pollinators have learned to take advantage of this phenomenon. The heliotropic plants track the sun to grow as abundantly and rapidly as they can in areas in which the reproduction cycle is short or limited due to environmental conditions. This hypothesis is prevalent for heliotropic species in the arctic climates.

\subsection{The Cooling Hypothesis}

The cooling hypothesis focuses on protecting plants from being scorched by the sun in hot climates. The heliotropic plants adjusts its position in reference to the sun to avoid overheating. This thermal regulation mechanism of the leaves enables the plant to control the amount of solar radiation incident to any given leaf [12]. In the hotter environments, a short growing season has led to adaptations that allow the heliotropic plants to thrive by absorbing as much light as possible and also the ability to protect itself once its optimal thermal range has been exceeded. This defense mechanism has enabled heliotropic plants to cultivate an ideal reproduction climate in some of the harshest environmental conditions that are uninhabitable for most plant species who do not exhibit this phenomenon.

\subsection{Benefits of Heliotropism}

Regardless of the theory or hypothesis as to why plants have heliotropic abilities, it is a hybrid ability that takes advantage of all the symbiotic relationships in its environment. Heliotropism can be viewed as an evolutionary modification of the characteristics of a plant by manipulating its capabilities in order to thrive in any environmental condition. Heliotropism enables a plant to absorb more warmth and light. This then leads to a warm and welcoming environment for insects which leads to an increasing likelihood of pollination, reproduction, and seed germination. While at the same time, the opposite can be true. Heliotropism enables a plant to prevent overheating. Which will also lead to an increasing likelihood of pollination, reproduction, and seed germination [13].

Plants that face the sun reach higher internal temperatures and also retain water more rapidly on average than flowers that just face directly upward. The same can be said for flowers who face the sun versus flowers who orient away from the sun. Therefore, heliotropic plants are less sensitive to ambient temperature changes than non-tracking flowers because this ability enhances the plant's capacity to warm under cool conditions and heat up under cold conditions. This ability allows the plants to avoid heat or cold stresses and maintain energy to focus on photosynthesis. The synergistic effects of heliotropic plants enable it to adapt to various climates around the world [14].

A common and persistent theme to heliotropic plants is that they all thrive in harsh climates. These plants can do this through the use of their adaptive leaves/heads. The heliotropic movements regulate the amount of solar radiation incident on a leaf. Depending on the plant species or time of day this regulation may be maximizing or minimizing the amount of sunlight. On clear days, heliotropism can reduce total radiant energy on the leaf blade by $20-30 \%$. Also, heliotropic plants can control the factors that enable photosynthesis which leads to an increased photosynthesis rate ranging from 29 to $42 \%$ [15]. Therefore, these movements have a profound impact on plant energy efficiency, leaf and plant temperature, water efficiency, and most importantly photosynthesis efficiency. The regulation of all these factors gives these plants an advantage in growth, seed production, and reproduction when compared to plants with stationary leaves [8].

\section{Main Components and Factors}

The main components related to heliotropic plants relate to their ability to follow the sunlight during the course of a day. When heliotropic plants are closely examined a few, specific factors will be discussed. The sun comes up and sets every day and plants, specifically the sunflower understands this process. Sunflowers prepare for the morning sunlight by facing east during the nighttime hours and following the sun throughout the day as it sets in the west. The second factor is phototropism. Phototropism is the process that helps a plant focus its leaves in the direction of sunlight. This focusing of the plant to the sunlight promotes additional photosynthesis.

The path that the Earth takes around the sun on its axis exposes the surface of the planet to repetitive changes in quality and quantity of the sun light received. This has created an environment where many plants attempt to receive all the available sunlight 


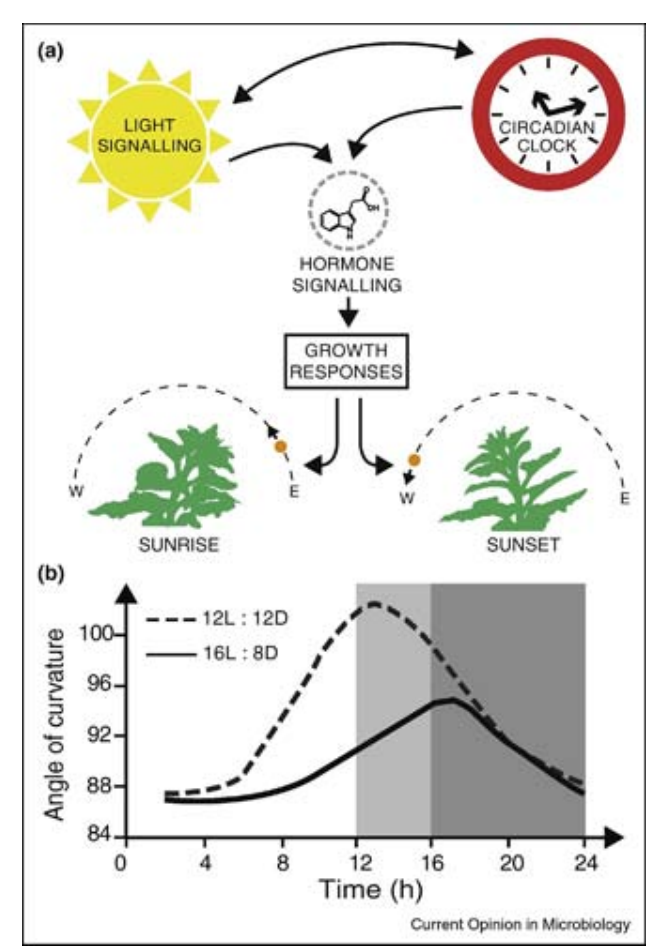

Figure 2: Sunflower growth affected by the circadian clock and sunlight [16].

provided by the sun for photosynthesis and healthy growth. The natural environment responds to these cyclic changes with rhythms in biological activity driven by a molecular pacemaker called the circadian clock [16]. Circadian clocks, as seen in Figure 2, are signaling networks that enhance an organism's relationship with the rhythmic environment, such as daylight and night coming each day. The plant circadian clock influences a wide range of physiological and biochemical events, such as photosynthesis and the induction of flowering [17]. This clock helps prepare plants to receive all available energy resources to support plant growth and pollination.

An additional growth pathway for plants is phototropism, this is a process in which plants align their photosynthetic organs with the direction of incoming light. This occurs when plants receive a light stimulus that then trigger growth in the cells furthest away from the light source below. This far side growth encourages plants to bend towards the light source to increase photosynthesis and healthy growth.

Heliotropism, or solar tracking, is a more dynamic form of phototropism, this generally occurs in the upper portions of the plant [19], [20]. Heliotropism requires a moving light source as plants grown in greenhouses or growth chambers with consistent over- head lighting do not display heliotropic movement, indicating that the behavior is dependent on a dynamic, directional light source [21]. Heliotropism is generated by the combined action of light-signaling pathways and the circadian clock. These two actions working in parallel enhance the plant's performance in the natural environment. Sunflower stems exhibit this movement such that their new growth on top shifts from facing east at dawn to facing west at dusk as they track the sun's relative position [22], [23], [24]. These additional pollinators promote healthy plants. As a sunflower reaches full growth it no longer follows the sun during the daytime and it faces east permanently [22]. By facing east the full size sunflower provides the warmest flower to the pollinator without "following" the sun throughout the day.

\section{Governing Physics and Parameters}

The governing physics and biology parameters associated with the plant's ability to maintain optimal sunlight through heliotropism has been established through evolutional changes of the plant in order to thrive in harsh environments. A heliotropic response is a sequence of the following processes: reception of the directional light signal, signal transduction, transformation of the signal to a physiological response, and the production of directional growth response [25]. These heliotropic processes and parameters of the plant have taken advantage of fundamental laws of physics which can be attributed to five common physics curriculum subjects. These topics focus on pressure, osmosis, light, spatial orientation, and surface area. The collaboration of these principles into one phenomenon has enabled plants to maintain an optimal amount of sunlight for the photosynthesis process.

\subsection{Pressure and Osmosis}

Pressure: the continuous physical force exerted on or against an object by something in contact with it and is defined as force per unit area [26].

Osmosis: a process by which molecules of a solvent tend to pass through a semipermeable membrane from a less concentrated solution into a more concentrated one, thus equalizing the concentrations on each side of the membrane [26]. 
When discussing heliotropic plants, the type of pressure used to move the plants is called turgor pressure. Heliotropic plants use this law of physics through its pulvinus organ. As mentioned earlier, the pulvinus organ is located at the base of the leaf or at the head of a heliotropic flower. The red lines in Figure 3 point out the pulvinus portions of a plant.

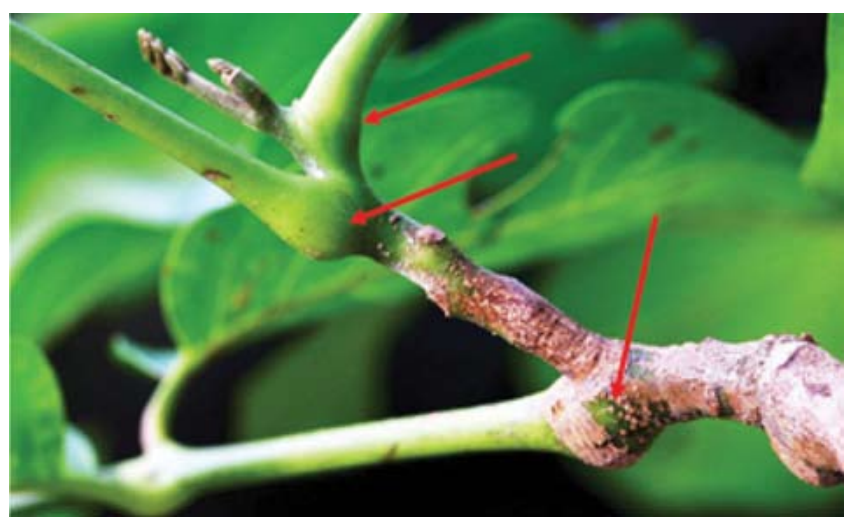

Figure 3: Pulvinus organs on a plant.

As seen above, the pulvinus organ is a joint-like thickening at the base of a plant leaf or flower head that facilitates the physical movement of the plant. They consist of a cavity of vascular tissue within a flexible, bulky cylinder of thin-walled cells [27]. Pulvinar movement is caused by changes in pressure leading to a contraction or expansion of the vascular tissue located inside the joint. This vascular tissue can be seen as the pockets of tissue indicated in Figure 4.

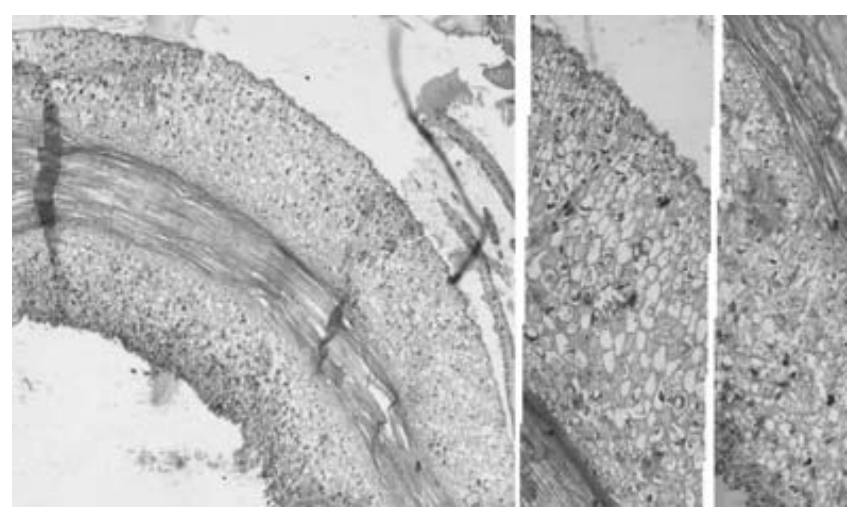

Figure 4: A depiction of the pulvinus organ and the plant's cell membrane [28].
The response is initiated when an increased amount of solute in the pulvinus decreases the water potential and triggers an efflux of potassium ions from the surrounding cells. This is followed by an efflux of water, resulting in a sudden change of turgor pressure in the cells of the pulvinus [28]. The process is a direct result of osmosis and can be seen in everyday processes like filtering water or the human's body's natural process of filtering blood [27]. Common examples of pulvinar movements include the night movement of legume leaves into a protective formation and the tracking of the sun by sunflowers throughout the day.

Turgor pressure can be envisioned as the internal pressure of a plant's cell wall depicted in Figure 5 below. Turgor pressure is a mechanism employed when the plant's plasma membrane is pushed against its cell walls. This pressure is caused by the flow of water from an area of low concentration outside the cell into the cell's cavity, which has a higher concentration [29].
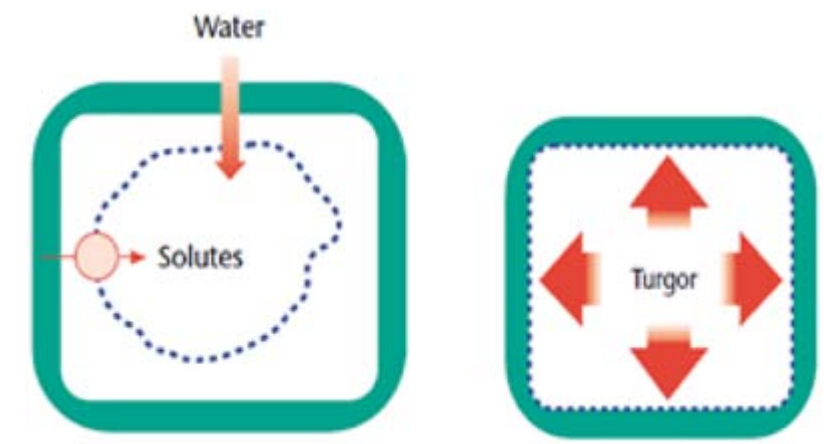

Figure 5: As water enters inside the cell wall indicated in blue through osmosis, the turgor pressure increases due to the cell's membrane enlargement [30].

The physical principle known as osmosis comes into play to cause the water to flow from one area of high water concentration and low solute to a low water concentration area and high solute. This process continues until the two areas have an equal ratio of solute to water. The cell's membrane permits the flow of water in and out of the cell but restricts the flow of solute.

Eventually, the cell's membrane is enlarged such that it pushes against the cell's wall. Changes in turgor pressure strongly depends on cell volume. The turgor pressure of heliotropic plants can be 
attributed to pressure seen in hydraulics and ratios seen in the osmosis process [31].

\subsection{Light}

Light: is defined as luminous, radiant energy in the form of electromagnetic radiation ranging in wavelength from 400 to $700 \mathrm{~nm}$ and propagated at a speed of 186,282 mi./sec, considered variously as a wave, corpuscular, or quantum phenomenon [26].

Light serves as the major energy resource to initiate the heliotropic process. Light's wavelengths trigger the heliotropic process through the perceived blue or red light by the photoreceptors near the major parallel veins. The photoreceptor molecules detect illumination and whether it is directed toward the tip or toward the base of the leaf. Whereas, the light perception by the pulvinus organ precludes signal transmission over a long distance. Thus, there are two different mechanisms for the detection of light direction in leaves: 1) a vein mechanism that senses light vectors 2) a pulvinar mechanism that senses light gradients [32].

Plants rely on several laws of physics in order to efficiently align the leaves to obtain the most/least amount of sunlight. Most heliotropic plants respond to the blue wavelength by aligning the leaves or flowers to an incident angle to the sun's wavelength. Whereas other plants whose goal is to protect itself from overheating will orient the leaves to reflect sunlight [33]. As seen in the Figure 6, the ray of light approaching the surface is known as the incident ray.

The ray of light that exits the surface is referred as the reflected ray. At the point of incidence, where the ray strikes the mirror, a normal line can be drawn perpendicular to the surface of the mirror The normal line serves as the median angle between the incident ray and the reflected ray. The angle between the incident ray and the normal is known as the angle of incidence. The angle between the reflected ray and the normal is known as the angle of reflection [34].

As stated earlier, an important factor which could seem obvious is the fact the light source must be dynamic in order for plants to exhibit heliotropic behavior. Scientists determined this behavior indicates heliotropism is dependent on a dynamic and

\section{Perpendicular to surface}

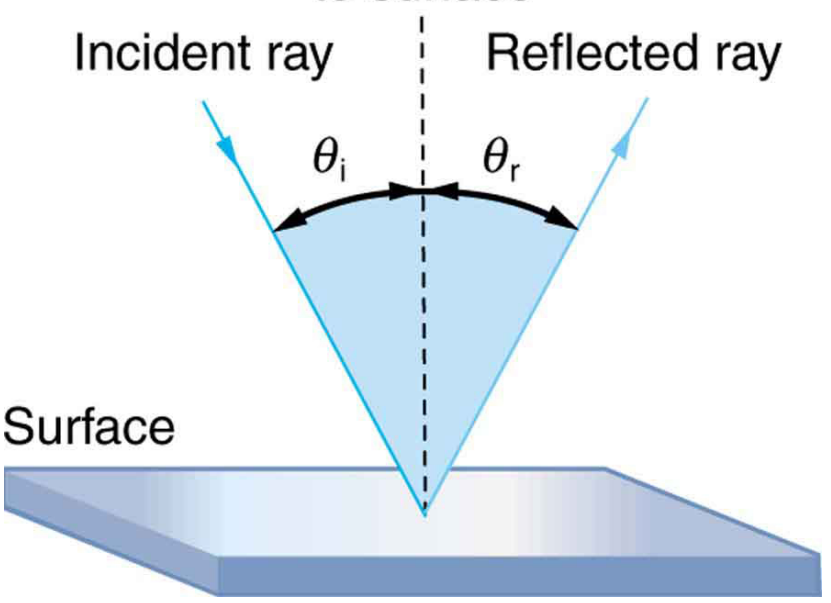

Figure 6: Laws of Reflection [34].

directional light source [35].The Plants orient their leaves in respect to these laws based on the purpose they are aiming to achieve. If the goal is to cool the plant, the leaves will orient to the angle of reflection to reduce the heating and may orient several leaves to offer shade in an orientation to reflect the sun's rays and heat. If the goal is obtain the most amount of sunlight the plant will track the sun and orient its leaves in the angle of incidence throughout the day to maximize the photosynthesis process. During the day, the sun warms the plants as waves of sunlight are absorbed and the thermal energy excites atoms in its leaves, triggering photosynthesis and creating heating. Too little heat results in very slow growth, but excess heat can be destructive. Plants have evolved and adapted by producing leaves with varying degrees of reflectiveness. Dark leaves absorb the most energy from sunlight, whereas light colored leaves reflect excess sunlight [36]. The plant's ability to change the angle of its leaves can reduce the total radiant energy on the leaf blade by $20-30 \%$. Therefore, these leaf movements have a large impact on leaf energy balance, leaf temperature, transpirational water loss, carbon gain through photosynthesis, and water use efficiency [37].

\subsection{Spatial Orientation and Surface Area}

Spatial Orientation: Defines the natural ability to maintain orientation and/or posture in relation to the surrounding environment (physical space) at rest and during motion [26].

Surface Area: measure of how much exposed area 
a solid object has, expressed in square units [26].

Plants use a combination of spatial orientation and the surface area of its leaves to ensure the maximum amount of sunlight can be absorbed or reflected through heliotropic movements. Up to now, the movement of individual leaves was analyzed. However, the spatial orientation and surface area of all the leaves in a plant's canopy has to analyzed to reflect the synergy of these aspects to form a systemized feature that enhances the plant's photosynthetic efficiency.

Scientists have determined that plants position its leaves so that each collects its share without interfering with any other leaf. This capability is achieved through multiple mechanisms of changing the posture of the leaves throughout the day as the sun moves across the sky [38]. Leaves can move in relation to each other on a non-interference basis by moving at greater and more varied amplitudes. Wide and ruffled blades prevent self-shading by spreading themselves apart and increasing their exposure to sunlight. This design allows the plant to control the amount of sun light it receives but also limit the amount of overheating. The leaves, through convective heat transfer, cool by wind blowing across the leaves [39].

If leaves overlap too much or are too closely spaced vertically, then the shading and light interference of the leaves will decrease the interception of the light rays. This will result in a decrease of photosynthetic efficiency. Conversely, if the arrangement of the leaves allow gaps in the canopy, then the photosynthetic efficiency will still decrease because the amount of sunlight will not be absorbed by the plant's leaves [40]. This arrangement can be seen in Figure 7.

Therefore, an equilibrium must be established between the amounts of space between each leaf in relation to each other. The shape of leaves also plays a big role in terms of the surface area. Curved shaped leaves offer small surface area for plants in hot climates. Whereas large leaves can capture as much sunlight as possible in areas in which the season is short. Thus, maximizing its photosynthetic efficiency in short seasons. To maximize cooling, some plants produce leaves with high surface area. Rough leaves have more surface area than smooth leaves. This provides more space for the transformation of liquid

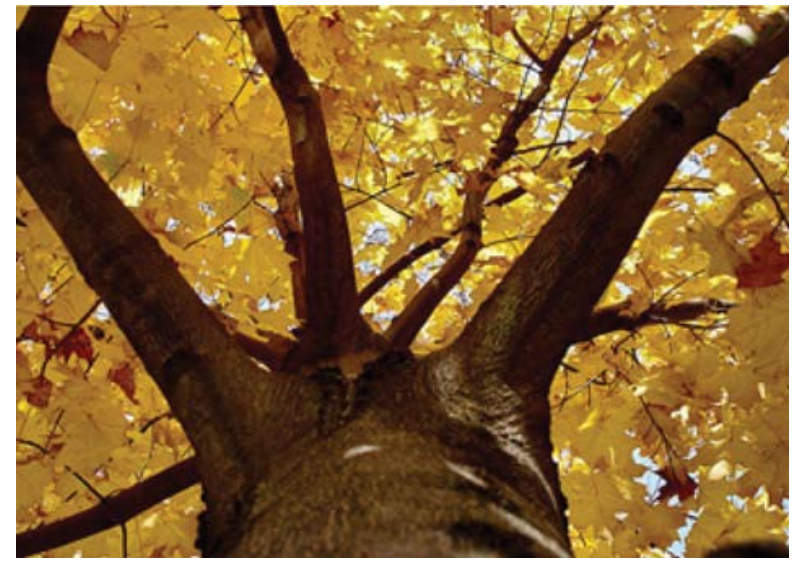

Figure 7: Spatial orientation of leaves in a Tree's canopy to minimize light interference or shade.

to vapor. As a result, there are greater cooling benefits due to the high humidity surrounding trees with rough leaves [41].

It is evident that the parameters associated with heliotropic plants relies heavily on the fundamental laws of physics and biology. The mastery of these laws has enabled the plant to tackle numerous stresses and climates. The heliotropic plant's ability to use pressure, osmosis, light, spatial orientation, and surface area to harness the sun's rays is indeed a phenomenon. The interaction of these elements and parameters, that when combined, produce a total effect that is greater than the sum of each of the individual leaf contributions. In effect, the plant has produced a redundancy to ensure its existence and reproduction. The duplication of all these critical components and functions as a system of efficient light absorbing antennas with the single intention of increasing its photosynthetic reliability has in a sense provided a backup or fail-safe to ensure system performance [42].

\section{Describing the Physics}

In a simplified manner, there are a few key concepts to grasp about how plants can and in fact do track sunlight. From the cycles of day and night to how the plant has the ability to attract additional pollinators by directing flowers and leaves towards a light source. The ability of nature to process sunlight into energy for the plant proves to be a complicated process. A simplified version of photosynthesis and heliotropism will be explored in the following sections. 


\subsection{Circadian Clock}

The path that the Earth takes around the sun on its axis exposes the surface of the planet to repetitive changes in quality and quantity of the sun light received. This has created an environment where many plants attempt to receive all the available sunlight provided by the sun for photosynthesis and healthy growth [43]. The natural environment responds to these cyclic changes with rhythms in biological activity driven by a molecular pacemaker called the circadian clock [16]. Circadian clocks are signaling networks that enhance an organism's relationship with the rhythmic environment, such as daylight and night coming each day. The plant circadian clock influences a wide range of physiological and biochemical events, such as photosynthesis and the induction of flowering [17]. This clock helps prepare plants to receive all available energy resources to support plant growth and pollination [44], [45].

Experiments conducted by Dodd have demonstrated that the circadian clock allows plants to increase photosynthesis. This occurs from the correct anticipation of dawn and dusk, and synchronization of the synthesis of light-harvesting complex proteins and chlorophyll [43]. Incorrect matching of natural rhythms to environmental rhythms reduced leaf chlorophyll content, reduced assimilation, reduced growth, and increased mortality [43]. Optimization of these parameters by circadian resonance has allowed plants to survive nature's evolution.

\subsection{Photosynthesis}

The word photosynthesis can be separated into two smaller words, "photo" which means light and "synthesis" which means putting things together. Plants require carbon dioxide $\left(\mathrm{CO}_{2}\right)$, water $\left(\mathrm{H}_{2} \mathrm{O}\right)$ and sunlight to produce their food. The plants put these together with sunlight to produce food and oxygen. Plants use this food for healthy growth, flowering and even producing fruit. Carbon dioxide from the air passes through small openings in the leaves called stomata. This carbon dioxide is combined with water absorbed by the roots of the plant and passes through vessels in the stem on its way to the leaves. Photosynthesis occurs in the cells of each leaf on the plant. Inside each cell there are small structures called chloroplasts. Each chloroplast contains a green chemical called chlorophyll which gives leaves their green color and absorbs the sun's energy. Eq.
(1) lays out what this process likes like in equation form.

$$
\begin{aligned}
& \text { Sunlight } \\
& 6 \mathrm{CO}_{2}+6 \mathrm{H}_{2} \mathrm{O} \rightarrow \mathrm{C}_{6} \mathrm{H}_{12} \mathrm{O}_{6}+6 \mathrm{O}_{2}(1) \\
& \text { Carbon Dioxide Water Glucose Oxygen }
\end{aligned}
$$

Photosynthesis uses the energy collected from the sun by the chlorophyll to split water molecules into hydrogen and oxygen. The remaining oxygen is then released by the leaves into the atmosphere. Some of the glucose that is created by this process is used to provide energy for growth and development of the plant while the rest is stored in leaves, roots or fruits for later use by the plants.

\subsection{Turgor Pressure}

Turgor pressure is critical to plant function as plants work like hydraulic machines moving fluids around. Turgor pressure helps with growth, transport and movement of fluids within the plant [44]. Plant cells generate hydrostatic pressures known as turgor pressure. The plants regulation of turgor pressure is the result of a plant's response to changes in nature, such as additional sunlight or temperature changes. A breakdown in this process of regulating the cell pressure reduces the performance of a plant. This breakdown occurs during stressful times of growth, such as limited water supply or extreme temperatures.

A major factor determining the magnitude of the turgor pressure is the total number of solutes accumulated inside the protoplast; the osmotic pressure of the cell contents $(\pi)$ provides a measure of this. The osmotic $(\pi)$ and turgor pressure $(\mathrm{P})$ of a cell are conveniently combined in the term water potential, which is a measure of the energy state of the water at any point [44]. Pritchard describes this equation as shown below in Eq. (2).

$$
\Psi_{w}=P-\pi
$$

The difference in water pressure between the cell and its environment are the driving force that determine if the cell takes on additional water. Water travels from a higher to a lower water potential to reach equilibrium. There are a number of factors can reduce turgor pressure below what is predicted but that is beyond what will be discussed here.

Cell extension drives nearly all plant growth and occurs because turgor pressure stretches the cell wall 
[46]. These forces generated by the cells can be very large, as can be seen by a plant's root system. A plant's root system can force its roots through compacted soil full of rocks and debris. Turgor pressure also provides a hydrostatic skeleton to support leaves and stems. This is evident when a plant wilts due to lack of water. Although plants are planted in one spot in the ground they can still manipulate themselves to gain a better incident angle with the sun rays. A plant's leaves and flowers move to track the sun, maximizing photosynthesis and pollination. In more extreme environments leaves turn away from the sun to prevent overheating. Such movements are caused by reversible variation in turgor on different sides of the stem.

\subsection{Heliotropism}

As stated throughout the paper, Heliotropism is a more dynamic form of phototropism, this generally occurs in the upper portions of the plant as it usually requires new growth to provide the directional change to "track" the sun. Heliotropism in the common sunflower is generated by the combined action of light-signaling pathways and the circadian clock. These two actions working in parallel enhance the plant's performance in the natural environment. Plants that experience heliotropism have been shown to provide about $9.5 \%$ increase in photosynthesis versus a stationary leaf [47]. This additional sunlight provides additional resources to the plant to promote healthy growth. Heliotropism is possible due to the growth of cells in a plant that are furthest from the light source. This growth promotes a directional growth towards the light source. Phototropism and heliotropism are complex biological responses involving interactions of multiple photoreceptors and multiple signaling pathways that together combine to produce a growth gradient on the shady side of a plant [48].

According to Shell, the ability of a plant to receive sunlight is given by the variable I, irradiance. This is the area of a leaf that is normal to the sun's rays. The larger this area the more significant the amount of sunlight the plant can process and is displayed as Eq. (3) [49].

$$
I=f_{i}(A) C_{i}
$$

Where $f_{i}(A)$ is the fraction of the total leaf area for a given plant and $C_{i}$ of $\cos \theta$ being the angle between the leaf-normal and the sun's beam. By using this calculation, it has been shown that the sunflower will intercept significantly more radiation than could be expected for a random leaf distribution. A random leaf distribution would be a distribution that does not follow any kind of process or sun tracking [49].

\section{Experiment and Analysis}

There are a few specific areas that could be focused in on for additional research. The areas of interest are listed below.

- Leaf Area Index

- Incident Angle / PFFD Transmission

- Leaf Temperature

- Turgor Pressure

\subsection{Leaf area Index}

Leaf area index can be calculated as seen below or it can be measured using a ceptometer. If this is measured, a reading can be taken in direct sunlight and compared to a reading taken directly under the canopy. These measurements can determine the health of a plant's canopy and be used as comparisons against past measurements. These measurements can also be used to determine optimal plant spacing and row directions during planting. This would be critical information to define in an effort to replicate this type of design in the engineering realm.

Thermal time or growth degree day (GDD) controls the amount of growth permitted by the plant. This comes down to the more sunlight the plant receives the more it can grow and produce. Therefore, testing the amount of sunlight each leaf receives or trying to optimize the amount of sunlight to each leaf will prove to be beneficial in this application. There is a balance between leaf density versus the size of the leaf. As the leaf gets larger the more of a shadow it will cast on the other leaves, therefore reducing photosynthesis in the other areas of the plant. Below are the calculations to determine the leaf area index for sunflowers. Growth degree (GD) is calculated from $\mathrm{Tb}$ and $\mathrm{Tc}$ being defined as base temperature and critical temperature respectively [50]. 


$$
\begin{aligned}
& G D=T_{b} \text { if } T<T b \\
& G D=T-T_{b} \text { if } T>T b \\
& G D=T_{c} \text { if } \quad G D>T c
\end{aligned}
$$

$\mathrm{T}$ is average daily temperature and growth degree day (GDD) was calculated by accumulating GD after the plant emerges from the surface. During the initial leaf growth rate, from emergence to the end of the vegetative stage, is referred to the period of fast linear growth during which the LAI increases at a constant rate and calculated by below in Eq. (4) [50]:

$$
\begin{aligned}
L A I(i)= & \left(L A I_{\max } / G D D 1\right) *(G D D(i)) \\
& \text { if } \quad G D D(i) \leq G D D 1
\end{aligned}
$$

Where $L A I_{\max }$ equals the $L A I$ at maximum growth rate and $G D D 1$ represents the accumulated thermal time from emergence till the end of the initial leaf growth rate [50].

The leaf area index in its simplest form is the amount of leaf area divided by the amount of ground surface area. This is a dimensionless number that can help gauge the health of a broad leaf plant's canopy.

\subsection{Incident Angle / Photosynthetic Photon Flux Density}

Incident angle is closely related to heliotropism in the fact that the plant follows the sun in an attempt to receive additional sunlight. The plant is trying to maintain a perpendicular angle to sunlight rays. Plants that experience heliotropism have been shown to provide an increase in photosynthesis versus a stationary leaf. This additional sunlight provides additional resources to the plant to promote healthy growth. Heliotropism is possible due to the growth of cells in a plant that are furthest from the light source. Phototropism and heliotropism are complex biological responses involving interactions of multiple photoreceptors and multiple signaling pathways that together combine to produce a growth gradient on the shady side of a plant [48].

According to Shell, the ability of a plant to receive sunlight is given by the variable I, irradiance. This is calculated by the area of a leaf that is normal to the sun's rays. The larger this area the more significant the amount of sunlight the plant can use to process carbon dioxide and water. This calculation is directly based on the incident angle of the leaf against the sun's rays.

The verification of this comes in the form of photosynthetic photon flux density (PPFD). This is the measurement of light (photons) that reaches the leaf. This measurement can be verified and tested with a ceptometer [51].

\subsection{Leaf Temperature}

Leaf temperature has an effect on how the plant behaves in relation to the sun. For example, a leaf that gets warmer than necessary will turn away from the sun to protect itself. This optimal temperature should be examined to help understand how nature solves this problem. This temperature safety feature that plants exhibit could be implemented in a slightly different way to provide safety to solar panels should a hail storm or extreme weather be coming. Understanding the temperature at which this happens on a plant will provide the details of how early this process starts and when the plant knows its time to start protecting itself. The test fixture pictured below in Figure 8 is how a test setup to measure the leaf temperature would be implemented.

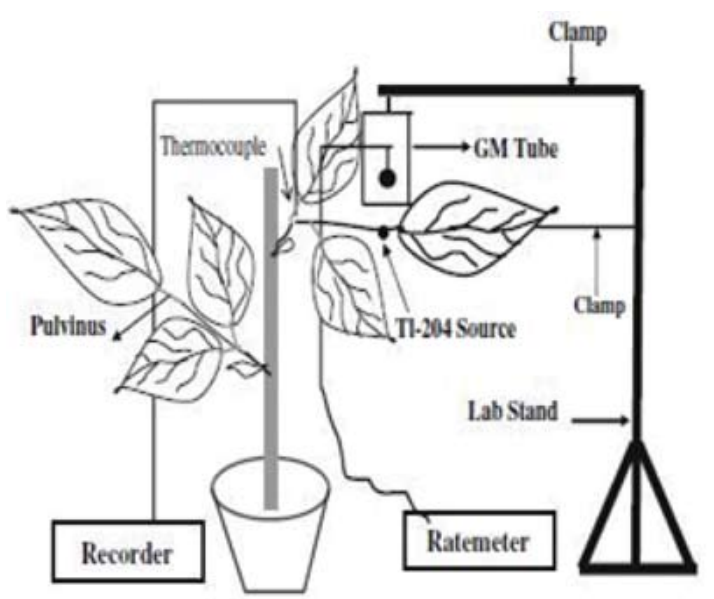

Figure 8: Setup to test leaf temperature [52].

\section{Feasibility, Limitations, and Advantages}




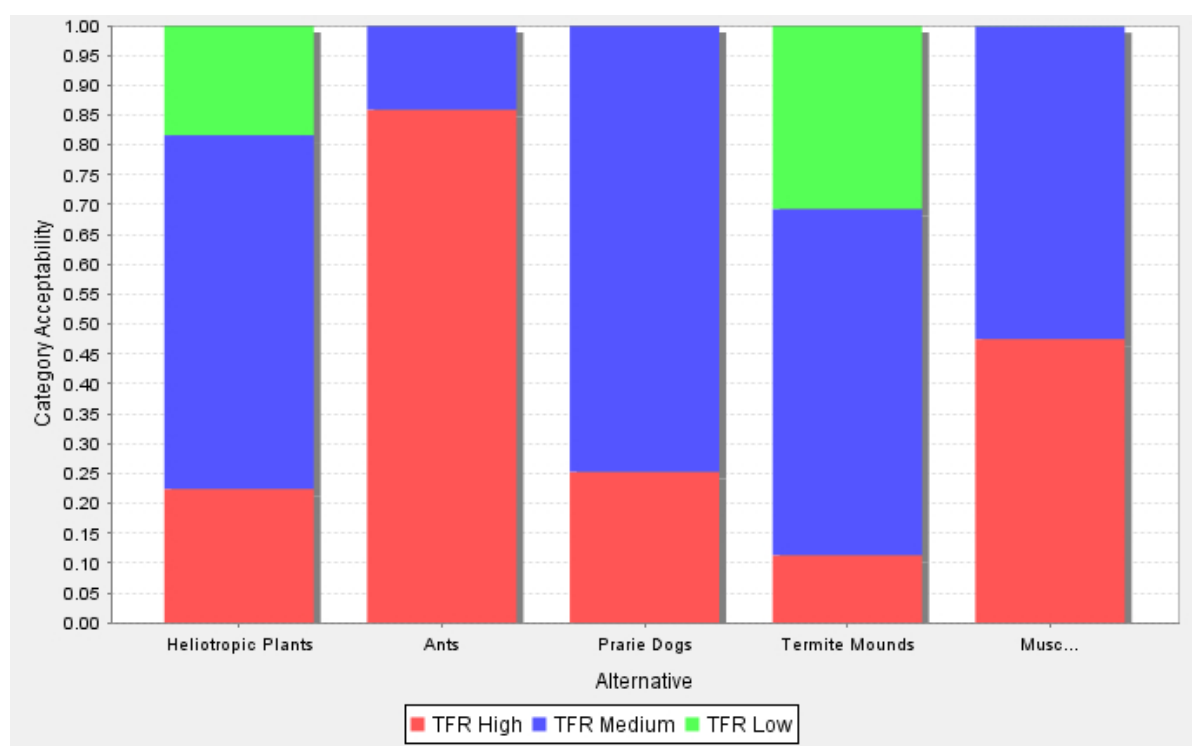

Figure 9: SMAA Results [53].

\subsection{Feasibility}

A team of four decision makers (3 Engineers, and 1 Biologist) were asked to complete the exercise using the Stochastic Multicriteria Acceptability Analysis (SMAA). The purpose of this exercise was to use SMAA with a set of criteria, derived from Biologically Inspired Design (BID), to assist multi-disciplinary decision makers evaluate biologyto-engineering transfer risk for candidate-design analogs. This framework guides decision makers to a low-risk choice by using externally valid predictors of design transfer risk [1]. The team decided to increase the number of decision makers to have a differing perspective that included considerations from a decision maker who was not an engineer. Also, the increase of decision makers allowed a stabilization of the standard deviation so that the results would not be hypersensitive to differing scores from 4 decision makers versus just two decision makers. The exercise utilized the design problem with the candidates and was completed in 3 rounds of scoring. The results are included for each round [53]. The results from the analysis are shown below in Figure 9.

The first and second round did not provide a definitive solution as no candidate was ranked over 0.1 in the TFR Low category. After each round, a clarification and discussion took place to help the decision makers align on a common thought process when analyzing the criteria and the ranking process. In the third round, two candidates emerged as the lowest risk options. The termite mounds and heliotropic plants proved to be the lowest design transfer risk to the engineering domain [53]. This analysis enabled us to pursue further.

\subsection{Limitations: Cost and Expertise}

The current team analyzing this phenomenon, with the intent of transferring this biological phenomenon to the engineering domain, is restricted by its disciplines and formal education in this broad and extensive scientific field. Biology is a complex field when trying to learn the underlying principles and assess if the biological solution is better than the existing ones available in today's market and technology. The ability to describe the complexity of this phenomenon is dependent on the approach. Each field of science and engineering defines and views complexity in different ways in the absence of a unifying concept or general complexity theory [54]. No one answer is correct and no one answer is wrong. However, a plant biologist would be able to uncover the most intricate components and processes to ensure no stone is left unturned. The expert would be able to offer additional biological principles that translate into the technological realm.

As of right now, the team consists of a mechanical and electrical engineer. In order to successfully implement a biomimetic engineering solution, a transdisciplinary perspective must be taken to ensure all aspects of the phenomenon are questioned, tested, and thoroughly examined. When solutions to complex problems are purposed, there are many facets 
of the solution that must be considered. Many times, these solutions must be considered through a transdisciplinary lens to examine the problem fully. The objective of transdisciplinary is to understand the present world, in all of its complexities, instead of focusing on one part of it [54]. While many solutions to complex problems have yet to satisfy all stakeholders, improved processes have been added to remedy the problems [55]. A complex problem that has not been addressed through transdisciplinary research and collaboration from all disciplines involved will produce a less than optimal solution [56].

Additionally, this biomimicry project would require the assistance of a statistician. One who would direct and frame the experiment and analysis portion of the project. The contribution of the statistical expert would focus on selecting and implementing the optimal analytic strategy and procedures for the data analysis of the experiments. Obtaining this resource would assist the team in collecting, inputting, and managing the data. Also, an expert would know the available software packages and tools that may be able to collaborate results, distributions, and theories. Understanding the various advanced statistical tests and models can correlate theories and conclusive evidence that may lead to an engineering innovation based on the behavior of heliotropic plants.

The cost to perform this research would be quite expensive due to our lack of statistical expertise. The experiment and analysis portion of our project focuses heavily on physical testing of the phenomenon and its processes. Utilizing simulations that could model the components/processes of the system and/or the phenomenon would greatly decrease the cost as well as offer a well-rounded analysis that could cover abundant iterations of the experiment. A cost-effective method in which the experiments can sample from the population with reasonable computational cost estimates based on a Monte Carlo Method would drastically improve the depth and robustness of the analysis and results while at the same time provide a path in achieving data with a high confidence interval [3].

\subsection{Advantages: Applicability of Circadian Clocks}

The applicability of circadian rhythms and clocks make this biomimicry project appealing and advantageous due to the immeasurable applications tied to circadian clocks. As mentioned earlier, circadian clocks have been traced to mammals, plants, fungi, bacteria and microorganisms and have a direct impact on each organism's health and behavior. Most people refer to the circadian clock as the biological or internal clock. Circadian clocks can be affected by natural and artificial light sources from the environment that cue influencing circadian rhythms to turn on or turn off genes that control an organism's internal clocks. For instance, the brain, kidney, liver, and hormonal system contain circadian clocks that must stay in sync in order to ensure efficient and healthy bodily functions in accordance with the time of day [57]. As a result of this, circadian synchronization within the cell and between organ systems is critical to health and well-being, and any breakdown of this time-based system can lead to harmful mental or physical conditions because circadian rhythms influence sleep-wake cycles, hormone release, body temperature and other important bodily functions. The interruption of this internal synchronization can lead to sleep disorders, obesity, diabetes, depression, bipolar disorder and seasonal affective disorder [57], [58].There are at least 15 genes that scientists believe make up the inner-makings of the circadian clock mechanism. Due to the variation of genetics from one person to the next, these components can result in profound differences in circadian clocks from person to person. This explains why some people are early birds and others are night owls [59]. Therefore, understanding how heliotropic plants correlate their movement to the circadian clock could offer insight to innovative applications or solutions in any or all of the following fields: medical, biomedical, biology, infectious diseases, pharmaceutical, and many more.

\subsection{Emerging Technologies}

\section{1) Solar Cells Mimicking Photosynthesis}

The emergence of existing technologies associated with Photosynthesis, Turgor Pressure, and Heliotropism make this topic advantageous to transdisciplinary and biomimetic engineers. Take for instance photosynthesis and its intended purpose of harvesting power from sunlight. Engineers have taken advantage of the photosynthesis phenomenon by replicating the harvesting of energy through the use of solar cells to produce electrical energy for humans. An extensive literature and market research of all the available technologies and products focused 
on solar cells led to the discovery of a solar cell design that utilizes the biological properties of the Ivy Plant. A company known as SMIT (Sustainably Minded Interactive Technology) designed their solar cells, as seen in Figure 10, to mimic the ivy plants by using Photovoltaic principles to convert wind and solar energy into electrical energy. Each 4 foot by 7 foot strip of the ivy leaves can generate up to 85 Watts of solar power.

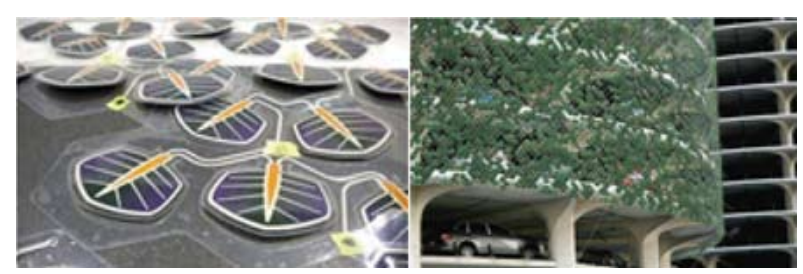

Figure 10: SMIT Solar ivy product.

This renewable energy will reduce heating and cooling costs while at the same time offer aesthetic and shading benefits to the structure [60]. The solar ivy also solves the major issue of non-aesthetic solar cells that take up large footprints of a building's roof. As seen in Figure 11, this product addresses this issue by utilizing a solution that can be installed on all the vertical sides of the structure that at the same time is aesthetically pleasing to the inhabitants of the building.

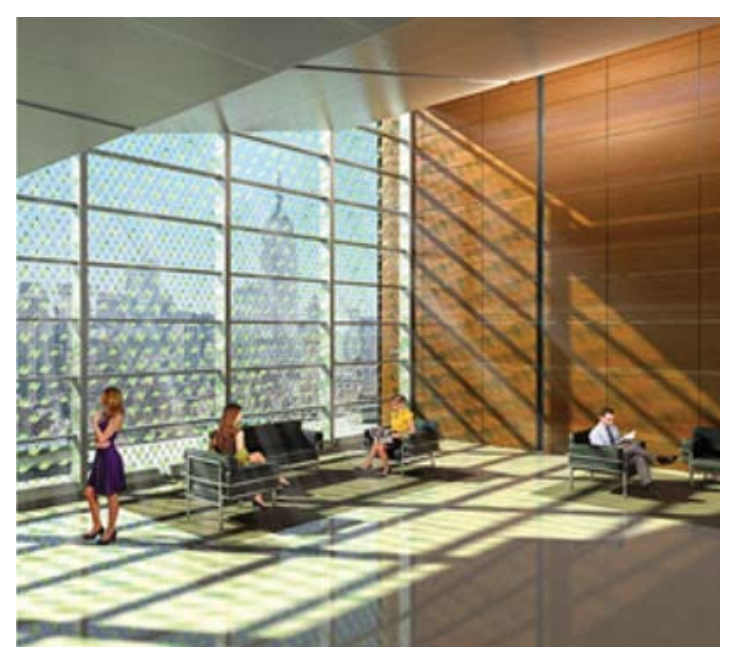

Figure 11: Residents view of the SMIT solar ivy product.
In addition to Photovoltaic Solar cells is another emerging solar cell technology focused on a dyesensitized solar cell. Dyesol has emerged at the forefront of this developing solar cell technology and market. The dye solar cell, as seen in Figure 12, is a low-cost alternative solar cell that is based on a semiconductor formed between a photo-sensitized anode and electrolyte. Compared to conventional silicon based photovoltaic technology, Dyesol's technology has lower cost and produces electricity more efficiently even in low light conditions and can be directly incorporated into buildings by replacing conventional glass panels rather than taking up roof or extra land area [61].

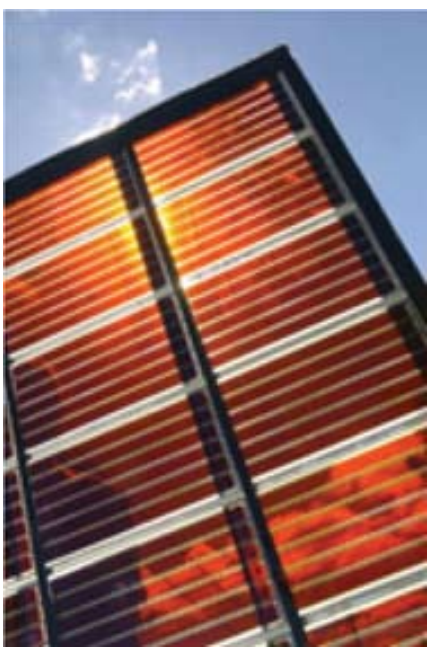

Figure 12: Dye solar cell.

Dyesol's latest product is the Perovskite solar cell which has a potential impact for the future of renewable energy because it is easy to produce and so flexible that it can be sprayed or painted on varying surfaces. Conventional Photovoltaic solar cells have peaked in regards to power conversion efficiency at a level of $25 \%$ for the last 15 years. Perovskite on the other hand is expected to reach the $30 \%$ efficiency mark within the next three years. This technology is already accredited to the current international standard IEC61646, which conducts temperature tests at 85 degrees Celsius for over a 1,000 hour period, in which solar cells cannot degrade in performance by any more than $10 \%[62]$. 


\section{2) Water Filtration Mimicking Heliotropic Cell Membranes}

In terms of Turgor Pressure and Osmosis, an emerging technology focused on water filtering relies on the fundamental laws discussed earlier in this paper. We can recall that turgor pressure relies on the osmosis process to transfer liquids across the plant membrane to achieve a desired level or equilibrium for the plant's pulvinar movement in order to track the sun. The Aquaporin Company has used biomimicry to mimic the cell's ability to transport water back and forth across membranes by way of a membrane cha" much like osmosis while at the same time filtering out particles or ions that are not particular to water [63]. This innovation has offered a new filtration solution in both a forward and reverse Osmosis process that can be employed in industrial and household applications. Discovery of the aquaporins has provided the explanation for selective transport of water across the plasma membranes of cells without allowing protons to pass. The Aquaporin Forward Osmosis (FO), as seen in Figure 13, offers an energy efficient product that can be applied to difficult wastewater streams.

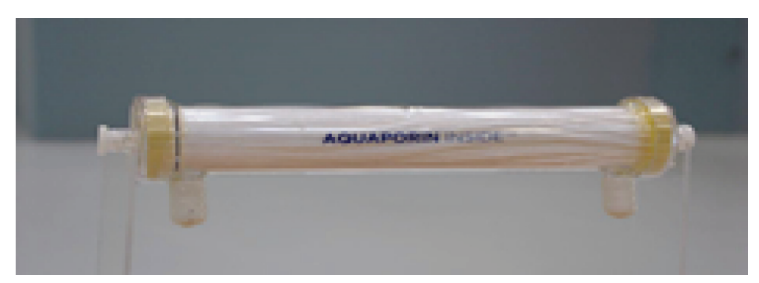

Figure 13: Aquaporin forward osmosis product.

The FO process uses only the osmotic pressure difference between a feed water stream and the draw solution as a driving force. The Reverse Osmosis (RO) product, as seen in Figure 14, processes polluted water into drinkable or reusable water.

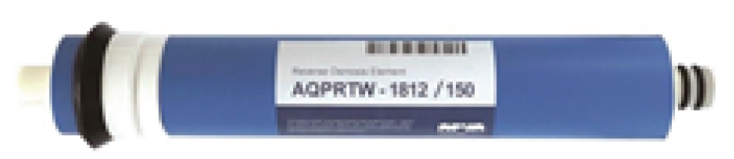

Figure 14: Aquaporin reverse osmosis product.
The Aquaporin technology allows RO membranes to have both improved water flux and higher rejection rates. Aquaporin is in the process of developing solutions for brackish water and seawater [64].

\section{Conclusion}

Irrespective of the limitations of our team, the transfer risk computed using the SMAA Tool indicated there is a low likelihood of risk in transferring this biological phenomena to the engineering domain. The Biomimetic Engineering goal is to create a product or process that can enhance society's capabilities. Using this approach to innovate a sustainable solution mimicking the plant's ability to maintain optimal sunlight through heliotropism to maximize the photosynthesis process would solve an unquantifiable number of current and future global issues.

The purpose of this paper was to offer an understanding of how plants are induced by sunlight as well as the long-term possibility of replicating this phenomenon for human purposes. All the questions presented in the introduction of this paper has led us to the fact that there are answers and solutions to the question, "Can the plant's heliotropism principles be translated by Biomimetic engineering to innovate or improve upon available technologies/strategies employed to harvest renewable energy?" By introducing a Biomimetic Engineering analysis of the plants' ability to maintain optimal sunlight in order to maximize the photosynthesis process, we have covered complex processes and parameters in multiple scientific and engineering subjects. The thorough analyzation provided in this paper covering the mechanics, physics, and features associated with heliotropism has led us to the conclusion that this phenomenon can be transferred to the engineering domain in a number of various applications and fields. An engineering replication of this biological phenomenon would ensure an innovative approach that would positively impact crop production, energy resources, water resources, medical/pharmaceutical solutions, pollution, energy production, and most importantly the lives of humans and organisms whose current long-term sustainability is often apprehensively analyzed due to the dwindling options of food and energy. 


\section{Acknowledgment}

We thank Dr. Burak Aksak of Texas Tech University for introducing us to the biomimicry field. The education and lecture enabled us to dive eagerly into this field and topic. Also, we are thankful for the support of Cortney Hines and Marissa Moore.

Funding: This research received no external funding.

Conflicts of Interest: The authors declare no conflict of interest.

\section{References}

[1] Williams M, Ertas A, Tate D. (2014). Using Stochastic Multicriteria Acceptability Analysis in Biologically Inspired Design as a Multidisciplinary Tool to Assess Biology-to-Engineering Transfer Risk for Candidate Analogs. ASME. J. Mech.Des, 136(11):111107-111107-7.

[2] https://biomimicry.org/what-is-biomimicry/ ( accessed April 21,2017)

[3] Aksak, B. (2017). "Biomimetic Class Lecture 1 Notes". Texas Tech University

[4] Chandler D. (2017). Leaf Group Ltd. www.sciencing.com/10fac tsonphotosynethesis ( accessed April 21,2017)

[5] MIT Nocera Lab Service RF. (2011). Artificial leaf turns sunlight into a cheap energy source. Science, 332 (6025): 25.

[6] Vandenbrink J, et al. (2014). Turning heads: The biology of solar tracking in sunflower. Plant Science, Volume 224, Pages 20-26, ISSN 0168-9452, http://dx.doi.org/10.1016/j.plantsci.2014.04.006.

[7] Cox, J. (2016). "Smarty Plants Despite their lack of brains, plants have exhibited a certain level of awareness in scientific tests". F + W Media

[8] Franke, L,et al. (2006). Heliotropism and water availability effects on flowering dynamics and seed production in Macroptilium lathyroides. Revista Brasileira de Sementes, Vol.28 (2), 45-52.

[9] Vancleave J. "Science fun Your Guide to Science Projects, Fun Experiments and Science Research" http://scienceprojectid easforkids.com/janicesscience-books/ (accessed April 21,2017)

[10] Hocking B.; Sharplin D. (1965). Flower basking by arctic insects. Nature, 206 (4980): 206215.

[11] Kevan, P.G. (1975). Sun-tracking solar furnaces in high arctic flowers: significance for pollination and insects. Science, 189 (4204): 723726.
[12] Lang A.R.G.; Begg J.E. (1979). Movements of Helianthus annuus leaves and heads. J Appl Ecol., 16: 299305.

[13] Proflowers. "How Do Flowers Follow the Sun? " http://www.proflowers.com/blog/how-do-flowersfollow-the-sun ( accessed April 21,2017)

[14] Galen, C. (2006). "Solar furnaces or swamp coolers: Costs and benefits of water use by solar-tracking flowers of the alpine snow buttercup, Ranunculus adoneus". Oecologia, 148(2), 195-201.

[15] Greer,D \& Thorpe, M. (2009). Leaf photosynthetic and solar-tracking responses of mallow, Malva parviflora, to photon flux density. Plant Physiology and Biochemistry, 47(10), 946-953.

[16] Yanovsky, M. (2017). Time to grow: circadian regulation of growth and metabolism in photosynthetic organisms. Current Opinion in Plant Biology, 35: 84-90.

[17] Hotta, C., et al. (2007). Modulation of environmental responses of plants by circadian clocks. Plant, cell \& environment, 30.3: 333-349.

[18] Briggs, W. (2014). Phototropism: some history, some puzzles, and a look ahead. Plant Physiol., 164, 1323.

[19] Vandenbrink, J. et al., (2016). Light and gravity signals synergize in modulating plant development. Front Plant Sci, vol. 5, 563. 117, 18.

[20] Briggs, W. (2016). How do sunflowers follow the Sun-and to what end? Science, 05 2016:Vol. 353, Issue 6299, pp. 541-542

[21] Lang,A. , \& Begg,J. (1979). Movements of Helianthus annuus leaves and heads. J. Appl. Ecol., $16,299305$.

[22] Schaffner, J. (1898). Observations on the nutation of Helianthus annuus. Bot. Gaz., 25, 395403.

[23] Shibaoka, H., \& Yamaki, T. (1959). Studies on the growth movement of the sunflower plant. Sci. Pap. Coll. Gen. Educ. Univ. Tokyo 9, 105126.

[24] Hamers, L. (2016). "Young sunflowers follow the sun's rays", Science News.

[25] Volkov, A. \& et al. (2004). Bioelectrochemical signaling in green plants induced by photosensory systems. Bioelectrochemistry, 63(1), 91-94.

[26] The Science Dictionary https://www.thescience diction nary.com ( accessed April 22,2017)

[27] Jackson, R., \& et al. (2008). The Cell. Biology (8th ed.). p. 134. ISBN 978-0-8053-6844-4.

[28] Kwan, K. et al., (2013). A Mathematical Model on Water Redistribution Mechanism of the Seismonastic Movement of Mimosa Pudica. Biophysical Journal, vol. 105, no. 1, 266-275, 2013/07/02/. 
[29] Steudle, E., \& Zimmermann, U. (2016). Effect of Turgor Pressure and Cell Size on the Wall Elasticity of Plant Cells. Plant Physiology, (170).

[30] Jordan, B., \& Dumais, J. (2010). Biomechanics of Plant Cell Growth. Encyclopedia of Life Sciences.

[31] Pritchard, J. (2001). Turgor Pressure. Encyclopedia of Life Sciences.Nature Publishing Group

[32] Donahue, R., Berg, V., \& Vogelmann, T. (1990). Assessment of the potential of the blue light gradient in soybean pulvini as a leaf orientation signal. Physiologia Plantarum, 79(4), 593-598.

[33] Galvão,V. \& Fankhauser, C. (2015). Sensing the light environment in plants: Photoreceptors and early signaling steps. Current Opinion in Neurobiology, 34, 46-53.

[34] OpenStax College. College Physics. https://courses.lumenlearning.com/physics/chapter/ 25-2-the-law-of-reflection/. ( accessed October $21,2017)$.

[35] Vogelmann, T., \& Bjorn, L. (1983). Response to directional light by leaves of a sun-tracking lupine Lupinus succulenttis. Physiol. Plant, 59: 533-538.

[36] Ehleringer, J, \& Forseth, I. (1980). "Solar tracking by plants". Science, 210, 1094.

[37] Attenborough, D. (1995). The private life of plants: A natural history of plant behavior. Princeton University Press.

[38] Greaver, T., \& Herbert, T. (2004). Heterogeneous reflected light influences asymmetry in leaf anatomy and gas exchang.Amer. J. Botany, 91: 1988-2003.

[39] Mahadevan, L., et al. (2008). How kelp produce blade shapes suited to different flow regimes: A new wrinkle.Integrative and Comparative Biology, 48(6), 834-851.

[40] Lin B., Lin Y .(2010). Cooling Effect of Shade Trees with Different Characteristics in a Subtropical Urban Park. Hortscience, 45(1):8386. 1 Department of Horticulture, National Taiwan University, No. 138, Sec. 4, Keelung Road, Da-an District, Taipei City 106, Taiwan.

[41] Duarte, L., et al (2013). Functional redundancy and stability in plant communities. Journal of Vegetation Science, 24(5), 963-974.

[42] Dodd, A., et al. (2005). Plant circadian clocks increase photosynthesis, growth, survival, and competitive advantage. Science, 309.5734: 630-633.

[43] Pritchard, J. (2001). Turgor Pressure Encyclopedia of Life Sciences. Nature Publishing Group, 1-3.

[44] Atamian, S., et al. (2016). Circadian regulation of sunflower heliotropism, floral orientation, and pollinator visits. Science, 353.6299: 587-590.
[45] Steudle, E, Zimmermann, U., \& Lüttge, U. (1977). Effect of turgor pressure and cell size on the wall elasticity of plant cells. Plant Physiology, 59(2), 285289.

[46] Kutschera, U., \& Briggs, W. (2016). Phototropic solar tracking in sunflower plants: an integrative perspective. Annals of botany, 117(1), 1-8.

[47] Ross, Ju. (1969). "Mathematical models of photosynthesis in a plant stand." Prediction and measurement of photosynthetic productivity. Proceedings of the IBP/PP Technical Meeting, Trebon,[Czechoslovakia], 14-21 . Wageningen: PUDOC, 1970.

[48] Atamian H, et al. (2016). Circadian regulation of sunflower heliotropism, floral orientation, and pollinator visits. Science, Vol. 353(6299), 587-590, DOI: 10.1126/science.aaf9793

[49] Shell, G., Lang, A., \& Sale, P. (1974). Quantitative measures of leaf orientation and heliotropic response in sunflower, bean, pepper and cucumber. Agricultural meteorology, 13(1), 25-37.

[50] Gholipouri, A. et al. (2009). Modeling Growth and Yield of Sunflower (Helianthus annus L.). Recent Research in Science and Technology, 1.5.

[51] Barradas, V., Jones, H. \& Clark, J. (1999). Leaf orientation and distribution in a Phaseolus vulgaris L. crop and their relation to light microclimate. International journal of biometeorology, 43(2), 6470.

[52] Raeini-Sarjaz, M., \& Chalavi, V. (2008). Pulvinus activity, leaf movement and leaf water-use efficiency of bush bean ( Phaseplus vulgaris L.) in a hot environment. International Journal of Biometeorology, $52(8), 815-822$.

[53] Moore, J, Hines, A, (2017). Bio-Inspired Design Lab. Texas Tech University

[54] Lu, S and Suh, N.P. (2009). Complexity in design of technical systems. CIRP Annuals - Manufacturing Technology 58.

[55] McGregor,S. (2006). Transformative Practice: New Pathways to Leadership. Kappa Omicron Nu Honor Society.

[56] Moore, J., Hines, A., Foreman, S. (2017). Transdisciplinary Design Process \& Systems Complexity Group Exercise 1. Texas Tech University.

[57] National Institute of General Medical Sciences, (2016). Circadian Rhythms Fact Sheet. https://www.nigms.nih.gov/education/pages/ Factsheet_CircadianRhythms.aspx ( accessed April $22,2017)$.

[58] Turek, F. W. (2016). Circadian clocks: Not your grandfather's clock. Science, 354(6315), 992-993. DOI: $10.1126 /$ science.aal2613 

[59] Duffield, G. (2017). Feeling tired for no rea-
son? Time to reset your circadian clock. Quartz https://qz.com/836292/what-is-a-circadianclock-and-how-do-you-change-it/ ( accessed April 21,2017).

[60] AskNature Team, The Biomimicry Institute (2015). "SMIT (Sustainably Minded Interactive Technology) Solar Ivy." from https://asknature.org/idea/smitsolar-ivy/\#.WNSOUYWcGIQ . ( accessed April 24,2017)

[61] AskNature Team, The Biomimicry Institute (2016). Dyesol Solar Cells.https://asknature.org/idea/dyesolarcelltechnology/\#.WP2 ESIWcHXh ( accessed April $24,2017)$.

[62] S3 Consortium Pty (2016) Ltd dye lays challenge tesla solar battle/http://www.nexttechstock.com/dyelays-challenge-tesla-solar-battle/ ( accessed April 24,2017).

[63] AskNature Team, The Biomimicry Institute. (2016). https://asknature.org/idea/aquaporin- membranetechnology/\#.WP2M-YWcHXg ( accessed April 24,2017).

[64] Aquaporin (2016) Products http://aquaporin.dk /product (accessed April 24,2017).

\section{About the Authors}

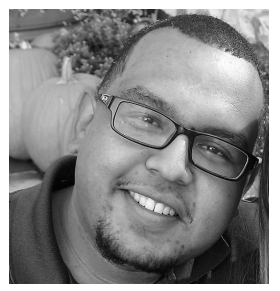

Jerome Moore has a BSEE from the University of Virginia and MSSE from Southern Methodist University. $\mathrm{He}$ is a Senior Systems Engineer at Raytheon and has been with the company for more than 10 years. He works in various roles within the Systems Engineering, Electromagnetic Environmental Effects, and TEMPEST technical fields. He is pursuing a Ph.D. degree through the Mechanical Engineering department at Texas Tech in Transdisciplinary Studies on Design, Process and Systems.

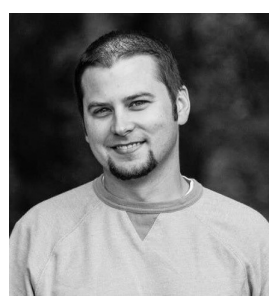

Adam Hines received a B.S. in Mechanical Engineering from the University of Texas at Arlington. He is currently a Senior Mechanical Engineer at Raytheon with 10 years of experience in electronic packaging and machine design. He is pursuing a master's degree through the Mechanical Engineering department at Texas Tech in Transdisciplinary Studies on Design, Process and Systems. 